Voix et Images

voixetimages

\title{
Jalons pour une narratologie du journal intime : le statut du récit dans le Journal d'Henriette Dessaulles
}

\section{Pierre Hébert}

Volume 13, numéro 1 (37), automne 1987

Suzanne Lamy

URI : https://id.erudit.org/iderudit/200691ar

DOI : https://doi.org/10.7202/200691ar

Aller au sommaire du numéro

Éditeur(s)

Université du Québec à Montréal

ISSN

0318-9201 (imprimé)

1705-933X (numérique)

Découvrir la revue

\section{Citer cet article}

Hébert, P. (1987). Jalons pour une narratologie du journal intime : le statut du récit dans le Journal d'Henriette Dessaulles. Voix et Images, 13(1), 140-156.

https://doi.org/10.7202/200691ar d'utilisation que vous pouvez consulter en ligne. 


\title{
Jalons pour une narratologie du journal intime: le statut du récit dans le Journal d'Henriette Dessaulles
}

\author{
par Pierre Hébert, Université de Toronto
}

Citant Arno Schmidt, Peter Boerner écrit ce qui a toutes les apparences d'un jugement sans appel: Le journal intime est une échappatoire pour les esprits confus, une des impasses de la littérature 1 . Cette attitude négative, à divers degrés acceptée comme prémisse du genre, pose ainsi d'entrée de jeu un objet flou de même qu'une impossibilité taxonomique. Un bon journal, écrit Kate O'Brien, doit être libre de la plupart des contraintes et exigences de l'oeuvre d'art ${ }^{2}$. Plus récemment, Béatrice Didier se rabattait sur le «je» comme seule règle d'un genre qui n'en connaît pas ${ }^{3}$. Comment dès lors classer ces objets amorphes, informes? Nombreuses sont les études qui procèdent par thèmes, par sujets traités: journaux de voyage, de guerre, de maladie, journaux spirituels, philosophiques, politiques, et il serait aisé de poursuivre cette énumération au point de la rendre inopérante: Les journaux, qui sont le reflet si fidele de la nature humaine sous toutes ses formes, ne sauraient être soumis à une classification strictet ${ }^{4}$. La seule tentative de classement qui ne procède pas par thèmes ou par analyse caractérologique (telle Michèle Leleu, dans les Journaux intimes, Paris, PUF, 1952), encore qu'elle fasse un usage plutôt métaphorique de concepts linguistiques et littéraires, est celle de Jacques Cocheyras, «la Place du journal intime dans une typologie des formes littéraires», dans le Journal intime et ses formes littéraires, p. 225-233. Ou encore parfois, c'est l'approche franchement externe qui prévaut: caractérologique, sociologique, psychologique.

On ne saurait trop insister sur les conséquences de ces a priori: faute d'avoir tenté de trouver une forme à l'informe, une ossature à l'invertébré, l'on ne dispose

1 Amo Schmidt, «Das Tagebuch und der Modern Autor», éd. Uwe Schultz, 1965, cité par Peter Boerner, «Place du journal intime dans la littérature moderne», dans le Journal intime et ses formes littéraires, textes réunis par V. Del Litto, Genève, Droz, 1978, p. 219.

2 Kate O'Brien, English Diaries and Journals, London, William Collins of London, 1943, p. 7 . Notons que toute citation tirée d'un ouvrage anglais est notre traduction.

3 Béatrice Didier, le Journal intime, Paris, PUF, 1976, p. 154.

4 P.A.Spalding, Self-Harvest: A Study of Diaries and the Diarist, London, Independent Press Ltd., 1949, p. 43. 
aujourd'hui d'aucune théorie du journal intime. Robert Fothergill a signalé cette carence en montrant que le diariste est d'abord engagé dans l'écriture d'un livre, et que le moi qui a préséance sur le moi révolu et le moi présent, c'est le moi écrit, le «moi textuel» si l'on veut. La question des procédés prend alors une importance nouvelle, voire première, si bien que l'idée selon laquelle toute technique ou stratégie d'écriture fait obstacle à la connaissance du «vrai moi» doit être rejetée. Un tel renversement ouvre la voie à la saisie d'un système textuel où tous les journaux intimes partagent certains traits communs: Fothergill parle d'ailleurs d'un common framework propre aux journaux où il faut établir une distinction mentale entre la première personne qui parle dans le journal et l'être historique qui tient la plume $[. . .]^{5}$. Il est intéressant de noter que la théorie du journal intime en est à établir des distinctions que la narratologie du récit a posées il y a de cela près d'une vingtaine d'années. Toutefois, Fothergill n'exploite pas à fond cette distinction pourtant fondamentale, et son étude se dirige ensuite vers les «attitudes littéraires» des diaristes. Béatrice Didier usera de la même modestie théorique, se contentant de relever que le moi qui écrit ne saurait se confondre avec l'homme, pas plus que le romancier ne se confond avec l'individu qui écrit le roman' 6.

Un point nouveau, pourtant: Didier établit, incidemment, une analogie entre le journal intime et le roman, en ce qui a trait à l'instance narrative. Jusqu'où peut-on pousser ce rapprochement? Le système textuel du journal intime participe-t-il des catégories habituellement dévolues au roman: histoire/discours, temps, mode, voix, etc.? À vrai dire, la question qui se pose ici rappelle celle qui fut soulevée il y a près de vingt ans par Roland Barthes: par où commencer? Notre but étant à la fois de poser le problème de l'existence du récit dans le journal intime et d'en proposer une méthode de description, nous commencerons par examiner le statut du récit dans le genre qui nous intéresse ici. Ce statut défini, nous pourrons ensuite articuler les catégories de base du journal intime dans lesquelles s'insère le récit; nous serons ainsi conduit à proposer une procédure de description illustrée à l'aide d'exemples tirés du Journal d'Henriette Desssaulles?. Le moment est d'ailleurs venu de définir les principaux termes que nous allons employer. Encore qu'il y ait peu de confusion possible à ce sujet, disons que par journal intime, nous entendrons la relation journalière ou périodique, mais de toute façon assez régulière, d'événements extérieurs, d'actions, d'expériences ou d'impressions personnelles 8 . Quant au terme diariste, désignant l'auteur d'un journal, il semble désormais consacré par l'usage au point qu'il ne soit plus nécessaire de le justifier. Cependant, par sujet du journal, nous

5 Robert Fothergill, Private Chronicles. A Study of English Diaries, London, Oxford University Press, 1974, p. 48.

6 Béatrice Didier, op. cit., p. 116.

7 Fadette. Journal d'Henriette Dessaulles, 1874-1880, Montréal, Hurtubise HMH, 1971. Désormais, toutes les références à ce journal seront indiquées dans le texte.

8 Elixabeth Bourcier, les Journaux privés en Angleterre de 1600 à 1660, Paris, Publications de la Sorbonne, 1976, p. 3. 
entendrons la représentation textuelle du diariste, ce sujet pouvant, selon les rôles narratifs, se manifester comme narrateur et/ou comme personnage. Par récit, que nous examinerons en détail plus loin, nous désignons la teneur événementielle du journal, son contenu narratif, proche en cela de son synonyme histoire ${ }^{9}$. Enfin, les propositions qui vont suivre, on s'en rendra compte, ne vaudront que pour le journal interne, selon la distinction qui remonte à Georges Gusdorf 10 .

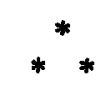

\section{Le statut du récit dans le journal}

Le point de départ le plus opportun pour poser la question du récit est, mutatis mutandis, celui-là même que fournit la narratologie. La distinction première, en cette matière, est celle du discours et de l'histoire (Bremond, Barthes, Todorov), de la narration, de l'histoire et du discours narratif (Genette). Que ces catégories soient binaires ou ternaires importe moins que leur point commun: l'existence d'une histoire, c'est-à-dire d'une succession d'événements, prise en charge par un discours, performance relevant d'un narrateur et impliquant un narrataire. Cependant, ces catégories élémentaires ne se présentent pas toujours aussi distinctement dans le tissu textuel et, qui plus est, font l'objet, depuis quelques années, de sérieuses remises en question. En effet, s'il est indubitable que le discours narratif a comme objet l'histoire, est-il tout aussi juste de considérer cette histoire comme antérieure à son existence discursive? Croire, comme l'ont fait Propp, Bremond, Todorov11, qu'il existe «réellement» une histoire antérieure à sa manifestation, n'est-ce pas jouer un jeu qui tient de l'idéalisme platonicien en l'occurrence des plus contraires à la réalité littéraire: l'idée et la réalité, l'histoire et le discours narratif? Car l'histoire n'existe que dans son incarnation discursive, ce que proposent avec une certaine hésitation Jonathan Culler12 et avec fermeté Barbara Herrnstein-Smith: selon elle, le

9 Nous hésitons cependant à considérer histoire et récit comme synonymes parfaits, dans le contexte où nous travaillons présentement: La raison en est que l'histoire renvoie le plus souvent à un modèle logique narratif préexistant au discours, alors que pour nous, dans le cas du journal, il nous apparaîtra impossible de séparer le contenu narratif du texte qui le prend en charge. Le mot récit veut refléter cette indissociabilité de Thistoire et du discours.

10 Georges Gusdorf, la Découverte de soi, Paris, PUF, 1948, p. 39 et suiv. Dans le journal exteme, l'événement compte plus que l'homme (p. 41), alors que le journal interne pose la chronique des événements dans le cadre de l'actualité personnelle, intérieure du diariste. Mais ce sont là des cadres a priori et notre façon d'aborder les journaux devrait permettre d'utiliser les concepts d'externe et d'interne comme point d'arrivée plutôt que comme point de départ.

11 Tzvetan Todorov, en particulier dans Communications 8, 1966.

12 Dans The Pursult of Signs. Semiotics, Literature, Deconstruction, «Story and Discourse in the Analysis of Narrative», Ithaca, Cornell University Press, 1981, p. 169-187. 
dualisme histoire/discours est une aberration méthodologique. Percevoir l'histoire comme une structure abstraite, c'est la considérer comme la re-présentation d'unités discrètes anté-discursives et, ainsi, s'interdire de la saisir comme un acte en fonction des conditions dans lesquelles il a été exécuté.

Ces considérations sur le statut de l'histoire dans ses rapports avec le discours ne sont éloignées qu'en apparence du journal intime, car elles nous permettent de poser d'une manière nouvelle nos questions de départ: le journal raconte-t-il quelque chose? le cas échéant, comment saisir le narratif dans le discours du journal?

Constatons cependant d'entrée de jeu que le caractère narratif du journal est à peu près universellement rejeté. L'une des considérations les plus généralement acceptées du journal intime, écrit Porter Abbott, [...] est qu'il est nécessairement sans forme et presque entièrement sans intrigue [plot] ${ }^{13}$. Tout en distinguant entre discours et récit, Béatrice Didier partage la même visée:

À priori ce genre se définirait par une absence presque totale de structure. Pas de elogique du récit», comparable à celle qui existe dans le conte ou dans le roman. Pour une raison bien évidente: il n'y a pas vraiment de récit. Et, curieusement, le journal diffère, en ce point, de l'autobiographie où l'on pourrait, du moins avec une certaine prudence, parler de récit. L'autobiographie est un récit construit après coup. [...] On pourrait aussi parler d'une logique du récit dans le roman autobiographique si peu chargé d'événements soit-il [...]. Il y a une logique du récit dans Adolphe, il n'y en a point dans le Journal de Benjamin Constant 14.

Chez Didier, la notion de récit se fonde clairement sur une logique de l'après coup, sur l'antériorité de l'histoire sur le discours. Pourtant, rien n'est moins certain: lorsqu'on observe de près la logique événementielle, chez Bremond par exemple, ne peut-on dire que tout comportement humain, dans son déroulement même, est récit? Les décisions prises, les pactes conclus, les déplacements effectués, ne peuvent-ils pas se laisser lire comme récit au moment même de leur manifestation? «L'après-coup» n'est peut-être, en définitive, que la construction de l'observateur qui n'a rien à voir avec le récit lui-même. Hors du discours, point de récit.

Voilà pourquoi le récit dans un journal - si récit il y a - conservera le repère de succession d'événements d'intérêt humain dans l'unité d'une même action mais que, surtout, il ne pourra être saisi que dans sa manifestation discursive, jamais en dehors, et surtout pas antérieurement à celle-ci. Pour que

13 Dans «Letters to the Self: the Cloistered Vision in Nonretrospective Fiction», PMLA, 95, no 1, janvier 1980, p. 44.

14 Le Journal intime, p.140. 
l'histoire d'une vie soit possible, il faut justement le recul historique, écrit encore Didier: désormais, allons dans le sens contraire et disons que le journal intime, du point de vue narratif, sera l'histoire d'une vie se nouant et se dénouant au jour le jour. C'est sur ce point capital que le journal intime se distingue des genres «rétrospectifs»: ce n'est plus en amont qu'il faut chercher sa narrativité, mais en aval. Eric Marty tient à ce sujet des propos déterminants: il ne s'agit pas, comme dans la réminiscence, de pousser l'écriture, dans un mouvement à reculons, d rejoindre en arrière un événement passé, pour le restituer; mais a l'inverse, de hisser l'événement du jour en avant, vers le présent de l'écriture, et de l'y répéter $[\ldots]^{15}$. L'histoire ayant été considérée traditionnellement comme une manifestation antérieure consolidée dans l'après-coup discursif, l'on a trop longtemps cru que le journal était ainsi dénué de toute narrativité. Le diariste, écrivant le plus souvent pour lui-même, privế de l'envergure récapitulative de l'autobiographe, ne pouvait en principe rien raconter. Il est temps de revoir ces conceptions, pour effectuer une volte-face complète et considérer le récit du journal du point de vue de la proue. Car si la poupe tient le gouvernail, à partir de «l'événement du jour», c'est bien la proue qui pointe vers cet espace ouvert, souvent incertain; et c'est là qu'il faut chercher le récit du journal:

Il y a "du» dans tous les genres littéraires, et sans doute dans toutes les formes d'expression 16: celte affirmation de Tadié n'apporterait rien aux problèmes discutés ici si elle ne conduisait pas à une discussion plus serrée du «récit dans le journal» et à sa place dans les catégories générales de ce genre.

\section{Les catégories du journal et la position du récit}

Georges Gusdorf propose une distinction, dont on a déjà fait état, entre le journal interne et le journal externe, classement acceptable s'il s'exerce sur des textes qui se situent aux antipodes du genre, comme par exemple un journal de voyage et un journal spirituel; et même des textes comme le Journal du siège de Paris de Crémazie et le Journal de Saint-Denys Garneau, à première vue susceptibles d'être qualifiés respectivement d'externe et d'interne, posent problème. Dans celui de Saint-Denys Garneau plus particulièrement, l'oscillation entre l'externe et l'interne est constante et la frontière entre les deux n'est pas toujours évidente.

Il faut même aller plus loin: tout journal, même celui où la part de l'interne se fait maximale, contient de l'externe, c'est-à-dire des événements à partir desquels s'embraie la chronique de l'actualité intérieure. Il s'ensuit que le journal - tout journal, devrions-nous dire - s'articule sur deux niveaux, celui de «l'événement du jour» et celui des répercussions, des réactions intérieures engendrées par le premier niveau. Fothergill signale d'ailleurs ces deux strates en notant leur proportion relative selon le type de journal:

15 L'Écriture du jour. Le Journal d'André GIde,Paris, Seuil, 1985, p. 12.

16 Jean-Yves Tadié, le Récit poétique, Paris, PUF, 1978, p. 7. 
Deux sortes de formes discernables apparaissent immédiatement. L'une est le récit [narrative] des événements du jour servant de cadre auquel le diariste peut y rattacher tout ce qu'il est enclin à y mettre sous forme de réflexion, d'expression de sentiments, la création d'une atmosphère, et ainsi de suite. Deux sortes de faits composent le cadre - les faits extérieurs [...] et les faits de l'expérience intérieure [...]. L'importance relative de l'expérience intérieure et extérieure, leur relation. la nature et l'importance du "commentaire auctorial», tout cela varie énormément d'un journal a l'autre. Dans un journal particulier, cependant, la configuration de l'empreinte tend d̀ demeurer assez constante, ou à changer graduellement sur une période étendue, de telle sorte qu'il est possible d'identifier les caractéristiques d'une entrée «typique»17.

La justesse de cette observation ne se poursuit cependant pas jusque dans ses conséquences. Fothergill ajoute en effet que l'accent mis sur la deuxième strate, celle de l'expérience intérieure, confere au journal un aspect décousu: $A u$ lieu du développement linéaire d'une histoire (story), l'empreinte étale les aspects épars d'un cahier spicilège du moi $[. . .]^{18}$. Ici, tout en maintenant les deux niveaux de Fothergill, nous devons nous démarquer de ce demier sur trois points essentiels qui nous serviront de fondement méthodologique:

a. Dans ces deux dernières citations, Fothergill situe le récit au niveau des «faits extemes» alors que nous soutenons ici exactement le contraire: s'il y a récit dans le journal, c'est au niveau des faits internes qu'il faut l'appréhender. Le récit, établissait Bremond, réclame une implication d'intérêt humain 19. Quand une diariste comme Henriette Dessaulles écrit: Ma cousine s'est mariée ce matin et elle part pour l'Europe. Elle est bienheureuse (p. 45), il n'y a pas encore de récit, même si cela correspond à «l'événement du jour» chez Fothergill. Le récit apparaîtra quand cet événement se trouvera intégré dans l'expérience intérieure. Et Henriette Dessaulles d'enchaîner, à la suite de l'extrait qui vient d'être cité: je voudrais voyager, aller très loin dans les beaux pays dont les noms seuls me font rêver! (p. 45). Le récit prend la forme d'un souhait, d'un projet, de l'ouverture d'une éventualité.

b. Nous ne distinguerons pas, comme pour le roman par exemple, entre une logique ou une chronologie antérieure des faits, et leur mise en discours. Nous envisagerons plutôt le discours du journal comme seule catégorie directement accessible à l'analyse, à l'intérieur de laquelle nous poserons deux niveaux: celui de l'événement narré, connu du diariste, et celui du récit proprement dit, où se

17 Private Chronicles [...], p. 58.

18 Private Chronicles [...], p. 60-61.

19 Claude Bremond, «la Logique des possibles narratifs», Communications, no 8, 1966, p. 62. 
formulent une série de modalisations (projet, crainte, souhait, etc.) en rapport avec l'événement du premier niveau.

Quant au récit, et afin d'éviter de capituler devant son aspect souvent décousu, il s'avère nécessaire de le décrire méthodiquement, c'est-à-dire d'en identifier et d'en regrouper les diverses manifestations.

\section{La description du récit dans le journal}

Nous prendrons, pour construire ce modèle descriptif, trois exemples tirés du Journal d'Henriette Dessaulles. De ces cas proposés comme exemples apparaîtront 1) les niveaux de thème/thème; 2) le rôle des fonctions syntaxiques à l'intérieur du rhème; 3 ) les mouvements extensifs et récessifs du rhème.

Exemple 1 (11 septembre 1974)

Concours de style - lère [sic]

Orthographe - 2ème

Histoire-1ère

Géographie - 2ème

et ainsi de suite, excepté l'arithmétique, j'étais cinquième. J'ai ri de voir leurs mines allongées, mais je n'ai pas été vilaine, au contraire, j'ai été gentille pour me faire pardonner leur petite humiliation d'autant plus difficile à avaler qu'elles m'avaient traitée comme une enfant trop jeune pour leur classe.

Ce petit triomphe me laisse triste. J'aimerais mieux avoir mon coin. être une des dernières, et qu'on m'aime (p. 23).

La structure de ce premier exemple offre manifestement les deux niveaux, celui des faits externes (réussite scolaire et comportement subséquent), et celui des faits internes (tristesse et manque d'amour; souhait). Le style syncopé, l'utilisation du passé composé, caractérisent le niveau des événements; le second, celui des faits internes, recourt à l'indicatif et au conditionnel présents. Cependant, les appellations faits externes/faits internes risquent d'engendrer des malentendus, surtout en ce qui a trait aux faits internes. Nous proposons donc de les appeler désormais thème et rhème. On aurait tort de ne voir dans ces nouvelles dénominations que la traduction en concepts nouveaux de réalités anciennes. Car le recours aux concepts descriptifs de thème et de rhème appelle par ce fait même une redéfinition des niveaux qu'ils désignent. Le thème désigne le support de l'information, le donné $e^{20}$; quant au rhème, il représente par rapport

20 Robert Martin, Pour une logique du sens, Paris, PUF, 1983, p. 208. 
au thème ce qu'on en dit 21 . De toute évidence, l'utilisation analogique proposée ici s'écarte du niveau phrastique étudié par les linguistes; mais elle s'y rattache indirectement dans la mesure où, on l'a souvent fait remarquer, le récit peut être considéré comme l'expansion d'une phrase. Dans ce premier exemple donné plus haut, nous aurons donc une entrée, c'est-à-dire l'association d'un thème et de un ou de plusieurs rhèmes qui y sont rattachés:

Entrée 1: 1. thème: réussite scolaire

2. rhème: souhait d'être aimée

(''événement)

(le récit)

Il ne serait pas difficile - mais trop long ici - de montrer à l'aide d'autres exemples le système de différenciation du thème et du rhème, au moyen du temps des verbes, de la disposition des paragraphes, de l'irruption de la subjectivité sur le plan du rhème, dans le sens où l'entend Benveniste. Mais ce qui importe avant tout, c'est la possibilité de découper le journal en unités de base, les entrées, composées d'un thème, qui peut ne donner lieu à aucun rhème (comme dans le cas des journaux de voyage, entre autres), ou qui peut engendrer un ou plusieurs rhèmes (c'est-à-dire des rhèmes de nature différente comme, par exemple, la crainte suivie d'un souhait). L'essentiel, dans ce second cas, c'est l'ouverture créée par le rhème, à la suite de l'aspect fini du thème. Comment se produit cette ouverture sur le plan du rhème? Un deuxième exemple permet d'approfondir cette question.

\section{Exemple 2 (15 avril 1874)}

Journée nulle, ça ne vaut pas la peine d'en parler. J'ouvre mon cahier comme si j'allais y trouver du bon. C'est une illusion. Je n'ai rien à dire. Une masse de leçons à préparer mais pas d'intelligence pour le faire. Oh! lâche, lâche, amollie que je suis! Ne m'aideras-tu pas mon Dieu dêtre autre chose qu'une cire fondue. C'est ridicule et des ce soir il faudrait changer. Je me donne jusqu'a demain puis il faudra marcher coûte que coûte! (p. 66).

Le thème est manifeste: journée nulle, manque d'intelligence pour écrire et préparer les leçons. Quant au rhème, il fait l'objet d'une expansion qui nous amène maintenant à le décrire en recourant à de nouvelles catégories, les fonctions syntaxiques. Nous avons jusqu'à maintenant soutenu cette idée que s'il y a récit dans le journal, ce n'est pas au premier niveau, celui de l'événement du jour, mais plutôt au second plan où le diariste s'approprie l'événement du premier niveau pour l'intégrer dans un discours caracterisé par une conduite à tenir, dans le sens le plus large possible. Dans ce contexte, les propos d'Émile Benveniste

21 Gilles Thérien, «Sémiologie dù discours littéraire», Recherches sémiotiques, vol. 4, no 2,1984 , p. 164 . Ces concepts ne sont pas neufs, et c'est sans doute Denis Slatka qui les a popularisés dans «l'Ordre du texte», Etudes de linguistique appliquée, Paris, Didier, 1975, p. 30-42. 
sur les fonctions syntaxiques permettent une description plus précise du rhème. L'énonciateur, selon Benveniste, dispose d'un certain nombre de fonctions, l'interrogation, l'intimation et l'assertion, de même que toutes sortes de recours formels, appartenant au mode (optatif, subjonctif: attente, souhait, appréhension) ou relevant de la phraséologie (les modalisants comme "peut-être», etc.: incertitude, indécision) 22 . Le centre de cette question réside dans le mode, que Benveniste utilise à quelques reprises, et qui, selon John Lyons, se définit par rapport à une classe non marquée de phrases qui expriment de simples affirmations de fait, neutres quant à l'attitude du locuteur envers ce qu'il est en train de dire23. L'opposition qu'établit Lyons entre affirmations de fait/attitude du locuteur, entre non marqué/marqué donc, correspond à la distinction thème/rhème dans la mesure où, justement, le thème désigne le niveau non marqué et le thème le niveau où transparaît l'attitude du diariste vis-à-vis du thème de base. Le paradigme rhème/attitude du locuteur/marqué peut être considéré comme modal en ce qu'il représente une régulation de l'information (Genette) foumie par le thème non marqué. Il s'ensuit que le sujet du journal, une fois le thème posé, dispose d'un répertoire de fonctions qui modalisent le premier niveau. Ces modes, selon Lyons (et ils recoupent les fonctions syntaxiques de Benveniste), sont les suivants:

\section{Impératif;}

2. Interrogatif, qui n'est pas traditionnellement considéré comme un mode mais, d'un point de vue général, les phrases interrogatives sont clairement modales; et on peut les définir par des modalités supplémentaires qui indiquent ce que le locuteur attend24;

3. Autres modalités, chacune ayant des degrés:
a. souhait, intention;
b. nécessité, obligation;
c. certitude, possibilité.

Ce répertoire peut servir à désigner les principales interventions du sujet du journal par rapport à son thème donné. Le terme de fonction syntaxique, trop restreint, ne convient cependant plus pour caractériser ces modalisations; celui de mode, trop général, peut s'avérer ambigu. Dans le cadre du journal intime, il conviendra plutôt de parler de fonctions rhématiques, désignant non seulement les divers modes que nous venons de proposer, mais aussi d'autres interventions possibles visant à intégrer, dans la subjectivité du diariste, le donné thématique.

22 Emile Benveniste, Problèmes de linguistlque générale I, «'Appareil formel de l'énonciation", Paris, Gallimard, 1974, p. 84-85.

23 Linguistique générale. Introduction à la linguistique théorique, Paris, Larousse, 1970 , p. 235.

24 Ibid., p. 236. 
Le deuxième exemple du Journal d'Henriette Dessaulles exploite quelquesunes de ces fonction rhématiques: un interrogatif (ne m'aideras-tu...), un souhait (il faudrait changer), une intention (il faudra marcher). La mise à jour des fonctions nous ramène à notre propos central: le récit dans le journal. En quoi ces fonctions contribuent-elles à cerner la teneur narrative du texte? Il importe, pour aborder convenablement cette question, de comprendre avec justesse la nature et la portée des fonctions rhématiques. Celles-ci, constituant le noyau du récit, sèment une indétermination, une indécidabilité, une ouverture quant à la conduite du sujet et, en cela, créent le foyer de l'intérêt que peut offrir un journal intime. Quels sont les souhaits, les craintes, les projets du sujet? Comment ceux-ci se répondent-ils, comme en écho, tout au long du texte? C'est à travers les fonctions thématiques que cet enjeu humain - et narratif - nous parvient.

Cette création de l'intérêt dans le journal, à partir du rapport thème/rhème, se greffe par ailleurs au principe fondamental de la théorie de l'information: le prévisible communique la signification, l'imprévisible transmet l'information. Le lieu du prévisible, dans le journal, est le thème: le sujet y écrit ce qu'il sait déjà. Le lieu de l'inattendu se situe au niveau du thème. L'on a si souvent fait état du flou, de l'indéterminé, voire du chaotique du journal - le demier chapitre de l'étude de Béatrice Didier ne s'intitule-t-il pas «le Journal, forme ouverte»? qu'il est étonnant que cette voie ait été négligée et que, dans la foulée d'Eco, de Moles, l'indétermination du journal fondée sur le rapport entre la signification et l'information n'ait pas encore été véritablement exploitée. Si cette optique ne peut qu'être évoquée ici, il n'en demeure pas moins que le lieu d'inscription du récit dans le journal et, partant, de son intérêt, se situe non pas au niveau du connu, mais plutôt au plan de ce qui s'ajoute, comme acquisition nouvelle, à ce que le diariste sait déjà. Les fonctions rhématiques livrent donc l'indéterminé généré par le thème, et qui peut rayonner dans le joumal en un réseau de projets, de craintes, de souhaits.

Enfin, un rhème peut-il se laisser caractériser globalement? Un troisième exemple aidera à étudier cette question.

Exemple 3 (1er juin 1874)

Belle journée au bois. Beaucoup de plaisir avec Soeur Sainte-Cécile. Jos en raffole, je l'aime bien, mais modérément. Nous avons cueilli des fleurs et chanté et couru et pleinement senti que nous vivons. Que c'est bon! C'est ça qu'il me faut, aimer tout le monde et toutes les choses et tous les êtres et me sentir toujours unie d tout, jamais repoussée ou tenue d̀ distance (p. 51).

Le thème apparaît clairement, la «Belle journée au bois», transmis au moyen d'une phrase nominale et du passé composé comme temps de base. Le rhème, au présent, indique un souhait faible, ou une sorte de nécessité, le cas est difficile à trancher: C'est ça qu'il me faut. Il est intéressant de faire, en l'occurrence, deux remarques. La première porte sur la phrase Que c'est bon!, qui 
a un statut problématique. Elle ne relève ni du thème, à cause de l'utilisation du présent, ni du rhème, puisque nous associons à celui-ci les caractéristiques d'imprévisibilité et d'ouverture que déclenchent les fonctions qu'il contient. Si, encore une fois, ce problème ne peut être traité à fond ici, il n'est cependant pas inutile de signaler ces phases de transition entre le thème et le rhème. Ces phases ne sont certes pas obligatoires, mais elles apparaissent fréquemment. La deuxième remarque, toujours au sujet du troisième exemple, s'applique à la structure parallèle du thème et du rhème: une série de coordinations de part et d'autre. Nous avons cueilli... et chanté... et couru... et pleinement senti... au niveau du thème, aimer tout le monde et toutes les choses et tous les êtres et me sentir... au plan du rhème.

Ces considérations particulières risquent toutefois d'éloigner ce troisième exemple de la caractérisation globale du rhème. Pareille caractérisation peut être fort précieuse pour l'analyse des mouvements du récit dans le journal. Ainsi, le ton général qui prévaut dans le cas que nous examinons présentement est celui, très positif, de l'harmonie d'Henriette avec les personnes et les choses. Cette impression favorable contraste singulièrement avec celle du premier exemple: la tristesse malgré la réussite scolaire. Or, la caractérisation globale du rhème peut ainsi se faire en vertu des deux grands mouvements qu'André Niel attribue au récit: l'extension et la récession, correspondant à des succès et des revers 25 . Dans le cas du journal, le thème peut ainsi donner lieu à un rhème extensif, transmis le plus souvent par des fonctions de souhait, d'intention, etc.; en revanche, le rhème récessif présentera des fonctions de crainte, de possibilités inquiétantes. Certaines fonctions peuvent évidemment ressortir à l'une ou à l'autre caractérisation, comme l'interrogation par exemple. Enfin, rien n'interdit au rhème d'être doublement marqué, c'est-à-dire d'être successivement récessif et extensif (ou l'inverse). L'exemple du mariage de Caroline, que nous avons déjà cité, donne lieu à un premier rhème extensif (je voudrais voyager...) puis, face à l'ennui présent, à un second, récessif: je voudrais dormir deux mois... ou me changer en rat!. Or, puisque nous avons parlé de mouvements, nous proposons de nommer mouvements rhématiques ces deux possibles que désignent l'extension et la récession.

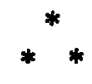

Cette étude du récit dans le journal nous a conduit à proposer une procédure de description qui fait appel à trois catégories conceptuelles: l'entrée, composée d'un thème et, le cas échéant, d'un rhème; les fonctions rhématiques, porteuses de l'information du rhème; les mouvements rhématiques, caractérisation globale sous les formes d'extension et de récession. L'étude des deux premiers cahiers 26

25 Andre Niel, «le Commentaire analytique», le Français dans le monde, no 75, septembre 1970 , p. 6-17

26 Le texte du Journal de Dessaulles pose ici un problème important de méthodologie: l'édition dont nous disposons actuellement, en attendant 
du Journal d'Henriette Dessaulles, selon les trois volets descriptifs, a été faite au moyen de l'encodage suivant (le $3^{e}$ exemple nous sert d'illustration):

\section{Entrées}

\begin{tabular}{|c|c|c|c|c|}
\hline \multirow[t]{2}{*}{ Thème } & \multirow[t]{2}{*}{ Liaison } & \multicolumn{3}{|c|}{ Rhème } \\
\hline & & Fonction(s) & Mouvement(s) & Objet(s) \\
\hline Joumée au bois & Exclamation & $\begin{array}{l}\text { Souhait } \\
\text { Nécessité }\end{array}$ & Extensif & $\begin{array}{l}\text { Aimer/ } \\
\text { être aimée }\end{array}$ \\
\hline
\end{tabular}

Pour la description du Journal, nous avons pratiqué ce codage linéairement; notre analyse ne tiendra cependant pas compte des liaisons, ni des objets des rhèmes, puisque nous voulons illustrer les seuls aspects que nous avons proposés en première partie, et qui fondent le récit dans le journal.

\section{Les entrées}

Ainsi codés, les deux premiers cahiers du Journal, des pages 21 a 237 , comprennent 139 entrées. L'étude de leur distribution peut être révélatrice; nous avons donc divisé les cahiers en tranches arbitraires de 10 pages chacune, ce qui fait apparaître la fréquence suivante:

$\begin{array}{llclc} & \begin{array}{l}\text { Cahier I } \\ \text { Pages }\end{array} & \text { Nombre d'entrées } & \begin{array}{l}\text { Cahier II } \\ \text { Pages }\end{array} & \text { Nombre d'entrées } \\ \text { 1. } & (21-30) & 18 & 12 .(133-142) & 12 \\ \text { 2. } & (31-40) & 11 & 13 .(143-152) & 9 \\ \text { 3. }(41-50) & 8 & 14 .(153-162) & 8 \\ \text { 4. }(51-60) & 6 & 15 .(163-172) & 2 \\ \text { 5. }(61--70) & 7 & 16 .(173-182) & 8 \\ \text { 6. }(71-80) & 6 & 17 .(183-192) & 5 \\ \text { 7. }(81-90) & 4 & 18 .(193-202) & 8 \\ \text { 8. }(91-100) & 5 & 19 .(203-212) & 3 \\ \text { 9. }(101-110) & 3 & 20 .(213-222) & 3 \\ \text { 10. }(111-120) & 1 & 21 .(223-232) & 5 \\ \text { 11. }(121-129) & 7 & 22 .(233-237) & 0\end{array}$

l'édition critique qui est actuellement en préparation, comprend quatre cahiers et, surtout, un hiatus de presque un an entre le deuxième et le troisième. Toute caractérisation globale, incluant les quatre cahiers, serait en vérité peu convaincante à cause de cette tranche manquante. Par conséquent, puisque notre but est tout autant de vérifier une méthodologie que de caractériser un texte, nous nous limiterons ici à ce qui forme un groupe homogène, les deux premiers cahiers. Nous préférons caractériser avec justesse une partie, plutôt que de décrire avec des lacunes la totalité. 
Manifestement, la plus haute fréquence apparaît au début de chaque cahier. Peut-on, cependant, considérer les deux cahiers pris ensemble? Il faudrait, à cette fin, accorder un statut particulier aux tranches 9 et 10: la baisse importante des entrées correspond au voyage d'Henriette à la mer: je vis dans un rêve (p. 110), écrit-elle, si bien que ces deux tranches méritent d'être considérées à part dans l'économie générale des deux cahiers. Elles se distinguent en effet doublement: par la disjonction spatiale de la narratrice et par l'installation d'un ton constamment positif dans le journal. Nous les mettrons provisoirement de côté, non sans en tirer l'enseignement qui s'impose: l'instauration d'un ton positif donne au journal une tout autre direction au point même, on le verra, de l'abolir.

L'allure générale de baisse des entrées c'est-à-dire, rappelons-le, des groupements thème/rhème, ne peut s'expliquer qu'en mettant l'accent sur les rhèmes, supports du récit et, plus précisément encore, sur les fonctions rhématiques ellesmêmes.

\section{Les fonctions rhématiques}

Avant de passer à l'étude des fonctions rhématiques, quelques distinctions nouvelles doivent être posées. Car ce serait manquer de discernement que d'accorder à toutes ces fonctions le même statut: certaines sont générées par un thème qui a pour objet les relations entre Henriette et Maurice, d'autres, entre Henriette et sa mère, et ainsi de suite. Or, comme nous traitons les rhèmes comme substrats du récit dans le journal, une précision exigée par l'étude de tout univers narratif doit être mise à contribution ici: l'établissement d'isotopies, de lignes d'action homogènes. Dans le Journal de Dessaulles, trois isotopies s'accaparent la majorité des fonctions hématiques:

a. les rapports Henriette-sa mère: 15 fonctions;

b. les rapports Henriette-Maurice: 46 fonctions;

c. les rapports Henriette vis-à-vis d'elle-même: 42 fonctions.

Voyons dès lors la configuration des fonctions rhématiques de ces trois isotopies afin de déterminer leur incidence sur la caractérisation générale de baisse des entrées qui marque les deux cahiers.

\section{a. Les fonctions rhématiques Henriette-sa mère}

Ces fonctions sont donc au nombre de 15; mais, plus que leur nombre, c'est leur distribution et leur regroupement en trois phases qui les caractérisent.

Pour ce qui est de leur distribution, 7 de ces fonctions se retrouvent aux 5 premières tranches du Journal. Mais, hormis cette répartition, leur nature impose un regroupement tripartite fort significatif. La première phase, aux tranches 1-5, présente des fonctions où Henriette souhaite aimer sa mère et être aimće par elle; la deuxième phase, aux tranches 11 et 12, se caractérise par un désir d'Henriette de se cuirasser, de ne plus souffrir de ce manque d'amour. Enfin, la troisième change radicalement: le souhait reste la fonction prédominante, mais l'objet 
differe. Ce qu'Henriette désire, ce n'est plus d'aimer ou d'être aimée, mais bien d'être bonne, de modifier elle-même son comportement. Ces trois temps peuvent être illustrés par ces trois fonctions rhématiques types:

1. La recherche d'une relation: [...] je voudrais qu'elle m'aime un peu. Oh! je voudrais, cet été, être gentille et affectueuse pour elle [...] (p. 55).

2. La recherche d'une attitude: je voudrais me cuirasser d'indifférence, ne plus sentir le mal qu'elle me fait (p. 128).

3. La recherche d'un comportement: je veux en finir avec mes petitesses (p. 186).

Les rapports d'Henriette avec sa mère méritent l'appellation de récit. En effet, le projet d'amour initial, qui se manifeste par la recherche d'un faire, se matérialise à la fin du deuxième cahier par la concrétisation de ce faire, de ce comportement vis-à-vis de sa mère. On notera en passant, dans ces trois fonctions types (et une étude lexicale de ce verbe dans les deux cahiers serait fort révélatrice), la mutation du verbe vouloir: je voudrais, je voudrais, je veux. Ce changement n'est pas l'effet du hasard, et nulle part mieux que dans cette prochaine citation Henriette n'a exprimé ce changement où elle passe de relations dysphoriques à une action concrète, à une positivité:

Je veux, il faut que ma conduite reflete ce pardon; c'est facile de dire: joublie, je pardonne. Je ne veux pas le dire, je veux le faire, je veux le vivre ce pardon et ça c'est difficile (p. 186; souligné dans le texte).

L'installation d'une positivité, d'une volonté est manifestée ici par la fonction d'intimation, et la prédominance du faire: l'importance de cette caractérisation narrative s'accroît avec l'étude de la deuxième isotopie, et deviendra la clef qui explique la baisse des entrées dans les deux cahiers.

\section{b. Les fonctions rhématiques Henriette-Maurice}

Il faut distinguer, vu le grand nombre de ces fonctions, quatre types: l'assertion, l'interrogation, le souhait et l'intimation.

L'assertion constitue une fonction rhématique dans la mesure où elle ouvre une indécidabilité, il faut le rappeler. Ainsi, elle se manifeste à l'occasion sous forme de prolepse. Dans le cas du Journal, les assertions se regroupent en trois temps:

- l'amitié: Oui, c'est lui mon ami (p. 25; aussi, p. 33);

- l'amour caché: [...] jamais je ne lui ferai d'aussi jolis serments (p. 151);

- l'amour à révéler: Saura-t-il jamais comme je l'aime (p. 226).

Le récit dans cette deuxième isotopie exprime une transformation complète chez Henriette en ce sens qu'elle s'avoue à elle-même son amour pour Maurice à 
partir de son amitié première. Et cette dernière phrase décisive - Saura-t-il jamais... - implique deux traits dynamiques essentiels à la poursuite du récit: une fin (la transmission de ce savoir) et un moyen. Cette double exigence créera à vrai dire une structure dialogique: Henriette veut révéler à Maurice son amour et, pour ce faire, lui donnera plus tard son journal à lire. Le journal permettra de communiquer ce savoir mais, on le verra, il sera par ce fait même détourné de sa mission première.

Les fonctions rhématiques interrogatives suivent ce même parcours de l'amitié à l'amour: Pourquoi me faire prier? (p. 32), quand Maurice réclame son portrait. Cette interrogation correspond à la phase d'amitié. Puis, l'indécision: J'aime Maurice, c'est bien certain, est-ce de l'amitié ou de l'amour? (p. 59). Ensuite, cet amour avoué dont Henriette mesure toute la grandeur: peut-elle aimer Maurice par-dessus tout? Impossible? Non, pas impossible, mais... (p. 62). Or, la zone de densité des interrogations se trouve bien sûr entre les tranches 4 et 14, ces moments où tente de se définir cet amour; au début, il s'agira d'amitié, ensuite, il faudra vivre cet amour. On pourrait d'ailleurs dire que les souhaits prennent en quelque sorte la relève des interrogations. Ces premiers se font plus nombreux à partir de la $16^{e}$ tranche $(6$ sur 12) et appuient cette positivité de la relation avec Maurice: leur objet est surtout de voir Maurice. En revanche, les intimations fléchissent à partir de ce même mouvement: elles avaient joué le rôle d'un frein (ne pas écrire à Maurice, se prouver qu'elle ne l'aime pas, etc.) qui devient inutile une fois l'amour avoué.

\section{c. Les relations d'Henriette par rapport à elle-même}

On retiendra trois classes de fonctions rhématiques: les interrogations, les souhaits et les intimations.

Les interrogations couvrent presque exclusivement le premier cahier. Jamais, à vrai dire, Henriette ne se demandera: Pourquoi, pourquoi tout ce triste de ma vie? (p. 48), dans le deuxième cahier. Ce rapport est justement l'inverse de celui des souhaits: très vagues dans le premier cahier, ils deviennent précis dans le deuxième. Prenons le cas du voyage: Je voudrais voyager, aller très loin dans les beaux pays dont les noms seuls me font rêver! (p. 45). Voyage sans objet fixe, si ce n'est une vague rêverie, qui se mue en recherche de l'autre au deuxième cahier: Si je pouvais voyager, m'ouvrir l'esprit, prendre contact avec le vraiment beau dans les beaux pays [...]! (p. 222). Cette transformation du souhait est importante; le souhait semble avoir trouvé un objet fixe, et il se caracatérise en l'occurrence par l'altérité.

C'est d'ailleurs cette idée de l'altérité qui marque la structure des intimations. Au début, il y a ce refus de l'autre: Et je ne veux rien quêter, jamais (p. 37). Cependant, un projet de réforme, si timide soit-il, s'impose chez Henriette: C'est le monde entier qu'il faudrait réformer. Quelle entreprise! Et si nous commencions l'oeuvre de réforme par toi (p. 136). Ce projet s'articule en volonté d'agir: il faut pouvoir sa vie (p. 183; souligné dans le texte), ce qui tourne Henriette 
vers l'autre, vers les autres: elle s'occupe des pauvres, veut causer avec d'autres de ses lectures: J'essaie de plus en plus de ne pas juger, d'entrer dans l'âme des autres pour tâcher de les comprendre (p. 214).

\section{Les mouvements rhématiques}

Afin d'expliquer la baisse des entrées dans les deux cahiers du Journal, lidée qui s'est imposée jusqu'à présent a été celle de l'installation d'une positivité, à travers les trois isotopies regroupant les fonctions thématiques. Cette positivité transparaît-elle dans les mouvements rhématiques?

En désignant par $\mathbf{E}$ et $\mathbf{R}$ les mouvements extensifs et récessifs des rhèmes, nous obtenons la configuration suivante, eu égard aux trois isotopies:

a. Henriette/mère

RR EE R E R EE R EE RR EEE

b. Henriette/Maurice

EEEEEEE R E R E R EE R E R EE RR E R EEEEE R E R EEEEE

c. Henriette/elle-même RRR E R EE RR EE RR EE E R EEEEEEEEEEE R E R EEEE

La positivité se manifeste sur ces trois plans. Ainsi, les rapports d'Henriette avec sa mère sont manifestement extensifs à partir de la deuxième moitié. Les rapports d'Henriette avec Maurice, extensifs lors des premières rencontres (lors de la phase d'amitié), deviennent mixtes, puis extensifs lors des 14 derniers mouvements (12 $\mathrm{E}$ pour $2 \mathrm{R})$. La troisième isotopie indique très clairement la prédominance des mouvements extensifs, dans la deuxième moitié. C'est ainsi que les mouvements rhématiques, aux trois niveaux isotopiques, se superposent dans un seul mouvement qui est en vérité celui des deux cahiers: l'établissement de la positivité dans ce qui représente un jeu à trois personnages, Henriette servant de lien commun.

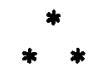

Au terme de cet article, la conclusion qui s'impose doit toucher deux volets: d'une part, le Journal de Dessaulles et, d'autre part, les limites mêmes de la procédure descriptive que nous avons proposée 27 .

La caractéristique dominante des deux cahiers, et qui en constitue le récit en même temps que les modulations de rapports entre les trois personnages, a été celle que nous avons désignée par «l'installation progressive de la positivité». Cette configuration générale apparaît dans le tableau synthèse suivant:

27 Il convient de signaler que cet article fait partie d'une étude en préparation sur le journal intime au Québec, en collaboration avec Marylin Baszczynski. L'ouvrage traitera de la réception du joumal intime, de l'évolution de la subjectivité, et présentera des études sur Dessaulles, Groulx et Saint-Denys Garneau. 


\section{Cahier I \\ 1. Entrées Baisse progressive de leur fréquence \\ 2. Fonctions rhématiques}

a. Henriette/mère Passage d'un souhait à un faire

b. Henriette/Maurice

Assertions d'amitié, puis d'amour, mais non révélé à Maurice

Interrogations sur la qualité

de cet amour

c. Henriette/elle-même

Interrogations sur le sens de

sa vie

Souhaits avec objets vagues

Intimations: refus d'autrui

Souhaits de voir Maurice

Souhaits tournés vers l'Autre

Intimations: dominer sa vie et altérité

Cette positivité du Journal se caractérise par deux traits: la prédominance de l'agir, aux trois strates isotopiques, et l'apparition de l'altérité. Il y aurait long à dire quant aux conséquences de ces changements sur le fait même d'écrire son journal pour Henriette Dessaulles. Car c'est précisément à cause de cette positivité que l'écriture du journal se trouve remise en question; à la fin du deuxième cahier apparaissent ces doutes, quand Henriette écrit: En cela, en tout moi, il y a de l'absurde, de l'extravagant, un excès de conscience, d'analyse qui me gêne et me nuit dans mes tendances révolutionnaires (p. 185). Cette révolution (pouvoir sa vie) s'accordera de plus en plus mal à l'activité qui consiste à tenir son journal. De même l'apparition de l'altérité détourne-t-elle le journal de sa fonction première. Ce jamais vous ne le verrez, ce miroir de moi (p. 185) perd de sa belle assurance quand Henriette, toujours à la fin du deuxième cahier, écrit: j'ai l'illusion [...] que je parle à Maurice, qu'il verra ces pages dans un lointain avenir (p. 198). Et c'est précisément à ce moment que le journal sera qualifié de manie (p. 198, 219). Henriette transmettra plus tard son journal à Maurice, et la condamnation définitive apparaîtra: Mon journal est devenu un ami un peu encombrant que j'aime toujours, mais dont l'utilité a cessé; c'est à Maurice que j'écris des pages et des pages [...] (p. 314).

Notons enfin que la méthode de description proposée ici, a l'instar de toute autre méthode, ne prétend pas avoir une allure définitive. Certaines limites la grèvent, dont particulièrement un manque de raffinement au niveau de la saisie des entrées, et du rapport avec ce que nous avons appelé le «técit» dans le journal et les autres instances du texte (narrateur, narrataire, etc.). De plus, l'analyse que nous avons proposée réclamait trop souvent la foi du lecteur, puisque la présentation de toutes les données eût été trop longue. Mais rappelons, à notre décharge, que nous l'avons proposée à titre d'exemple. Par ailleurs, il nous semble désormais acquis que le journal intime ouvre à l'analyse littéraire un vaste champ dont on n'a jusqu'à présent que circonscrit les limites, à la condition cependant de se débarrasser du préjugé d'informe qui en a empêché l'élaboration d'une théorie. 